\title{
Flexible Channel Selection Mechanism for Cognitive Radio Based Last Mile Smart Grid Communications
}

\author{
Saud Althunibat, Qi Wang, Fabrizio Granelli \\ DISI, University of Trento, Trento, Italy \\ althunibat@disi.unitn.it; wang@disi.unitn.it; granelli@disi.unitn.it
}

\begin{abstract}
Smart Grid (SG) operation requires a reliable, accurate and effective communication link between the distributed meters and the control center. However, dedicating a portion of the spectrum is difficult due to the spectrum scarcity problem. Cognitive Radio (CR) technology has been nominated as a good candidate for SG communications due to its efficiency and flexibility. Indeed, channel selection in CRbased SG systems is still an open issue, and it is investigated in this paper. The paper proposes a novel channel selection mechanism that is able to adapt the selection criteria based on the type of transmitted data. The proposed mechanism is proven to provide high performance compared to the non-adaptable mechanisms.
\end{abstract}

\section{Cognitive Radios For SMart GRid COMmunication}

The communications infrastructure for smart grid (SG) has recently received an increasing attention. Given the heterogeneity of the SG, it is necessary to clarify that the considered scenario in the paper consists of the communication links used to interconnect the distributed meters at the customers' side and the local control center. To some extent, from a communications perspective, this could be considered the last mile of the SG system. Many wireless technologies have been nominated in the literature as candidate infrastructures for SG communications in such scenario [1]. However, two main properties that may distinguish SG nature from other wireless systems: first, both the transmitter and receiver are always fixed, which alleviate many problems generated by mobile transmitters, such as fast channel variation, frequent handovers, ...etc; second, a considerable part of the transmitted data have a regular (fixed) generation rate, i.e., both transmitter and receiver know who will send these data and when. 
Moreover, such types of data usually tolerate a relatively long delay (in communication terms). These properties should be taken into account in order to identify suitable, efficient and reliable communication infrastructures for the SG.

Cognitive Radio (CR) technology is one of the candidate technologies to serve for last mile SG communications [2]. CR is an intelligent software-defined radio technology that facilitates efficient, reliable and dynamic use of the unused portions of the radio spectrum by adapting its configuration according to the environment radio conditions [3]. The IEEE 802.22 is the first standardization activity on CR networks based on opportunistic utilization of TV spectrum bands [4].

CR based on IEEE 802.22 represents a good candidate for last mile SG communications for several reasons, $i$ ) Unlike other wireless technologies, CRs operate in unused TV frequencies, which, in view of spectrum scarcity, represents an extremely useful feature. $i$ i) high data rates up to tens of Mbps can be achieved by CR. iii) Due to the long-range propagation characteristics of the TV bands, the coverage area can reach up to $100 \mathrm{KM}$. iv) $\mathrm{CR}$ is adaptive, programmable and flexible, since it is built on a software-defined radio platform.

Although the above mentioned reasons can make CR a preferred choice for SG communication, three main issues should be still addressed. First, identifying the unused TV frequencies represents a key issue in CR implementation. Usually, information about band occupancy is obtained by a pre-process called spectrum sensing, which consumes time and energy [5]. Second, errors can occur in identifying the availability of the TV bands due to probable imperfect spectrum sensing, which is reflected on the reliability of cognitive transmission [6]. Third, cognitive transmission is a threatened transmission due to the possible and unpredictable appearance of the original licensed user during transmission. However, the last issue becomes less significant in case of CR based on IEEE 802.22 since a TV frequency that has been identified as vacant cannot be reused during CR's usage in the same area. As a consequence, The former two issues (the first and the second) are taken into consideration throughout this work.

Based on the dynamic smart grid communication environment and comprehensive communication applications in the smart grid architecture, researchers have explored different challenges of the CRbased SG communications in recent years. A. Ghassemi, et al. [11] studied current energy management requirements in the smart grid and employed $\mathrm{CR}$ as the solution for the communication requirements in the wide area networks (WANs) of SG, based on IEEE 802.22 standard. In particular, the additional bandwidth required for non-critical data is addressed by [11]. Stand-alone radio and secondary radio were proposed for CR communication systems based on IEEE 802.22 standard. According to such two architectures, [11] employed dual-radio scheme for CR-based transmission, which can offer an effective spectrum sensing process.

The errors can occur during the process of spectrum sensing, maybe caused by dynamic parameters in the harsh SG environments, just as equipment noise, cross-tier interference and etc.. According to this 
challenge, Ghalib A. Shah, et al., [12] presented a CR-based cross-layer framework to meet the QoS requirements of diverse SG applications. The proposed mechanism employed the emerging cognitive radio technology to reduce the noisy and congested spectrum bands, yielding reliable and high capacity wireless links for SG communications. Meanwhile, a distributed control algorithm (DCA) was also designed to support QoS via channel and control, scheduling and routing decisions, which maximizes the network utilization under the constraints of QoS.

Due to the features of IEEE 802.22 standard, CR based communication technologies can serve the SG applications with high reliability even in harsh environmental conditions. The growing demands of multimedia applications in SG communications, require large bandwidth and network resources. In particular, large-size and time-sensitive multimedia delivery requires more reliability and stability in CR based SG communications. In this case, H.G. Wang et al. [13] and J.F. Huang et al. [14] explored related issues of CR based multimedia communications and delivered their own mechanisms. Considering the mutual interactions with the enhanced reliability and efficiency of whole SG system, H.G. Wang et al. [13] proposed a combined scheme of electrical and CR based networks to effectively support the wireless transmissions of large-sized multimedia data created by the dynamic SG applications. In order to achieve the required Quality of Experience (QoE) performance in the SG system, a CR networking paradigm is also proposed in [13] to efficiently manage the channel allocation for both primary users (PUs) and secondary users (SUs).

As we discussed previously, the CR based communication platform is essentially needed to support large-size multimedia data delivery in the SG environment. According to the various traffic types of SG communications, J.F. Huang et al. [14] developed CR-based channel allocation and traffic scheduling schemes to mitigate the risk in multimedia data transmission via employing CR based communication networks. In these schemes, two types of channel switch, including periodic switching and triggered switching are introduced. Meanwhile, the spectrum sensing errors are also considered for supporting such mechanisms. Based on the proposed schemes, the different traffic types in SG are prioritized for traffic scheduling of the SUs and the system utility optimization problem for SG communications system is solved as well.

In this paper, we focus on the channel selection in CR-based SG communications. Specifically, we propose a mechanism for selecting the best channel to use for SG communications. In the proposed mechanisms, the type of transmitted data plays a significant role in choosing the best channel, as the criteria for selecting the transmit channel changes according to the transmitted data. In detail, data that are delay sensitive should be transmitted through a channel with high reliability, while other types of data that are not delay sensitive can be transmitted over channels with high data rates. Based on such principle, the transmit node will have more freedom to select the transmit channel based on the special requirement for their instantaneous transmitted data. 
The rest of the paper is organized as follows. Section II presents the system model considered in this paper. The proposed mechanism is discussed in detail in Section III. The performance of the proposed mechanism is explored through simulation results in Section IV, and finally conclusions are drawn in Section V.

\section{System Model}

A typical SG system is considered, as shown in Fig. 1. In general, a SG consists of power plant, power utilities,transmission grid, distribution grid and terminal power consumers. There are two types of flows in the SG system, the power flow and the information flow. One of the important differences between the traditional power grid and the smart grid is that terminal power consumers do not only unilaterally receive the orders from utilities, but they can also report and send their demand information back to utilities via communication links. Considering the SG communications environmental conditions, safe, stable, reliable and efficient communications between terminal power consumers and utilities is recognized as a huge challenge.

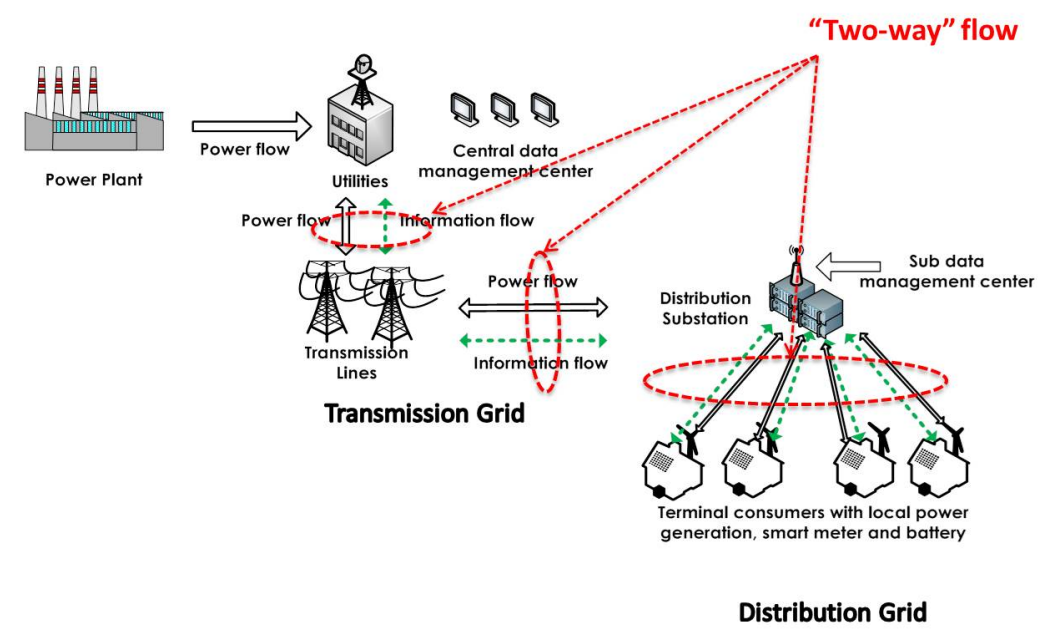

Fig. 1. A simplified view of a typical smart grid system.

Recently, the major changes are being promoted in the distribution networks of SG system. A simple architecture of the distribution grid is shown in Fig. 2. From Fig. 2, the distribution grid represents the last mile of the SG and it includes, primary substation, secondary substation, feed lines and terminal power consumers with residential renewable energy generation system. Huge amount of terminal power consumers would have dynamic and random power consumption behaviors that would generate an aggregate power demand and other potential requests to the utilities. The paper discusses the usage of the TV spectrum based CR communication technologies as a potential candidate to establish the wireless communication links in the distribution section of the SG system for the multimedia information delivery. 


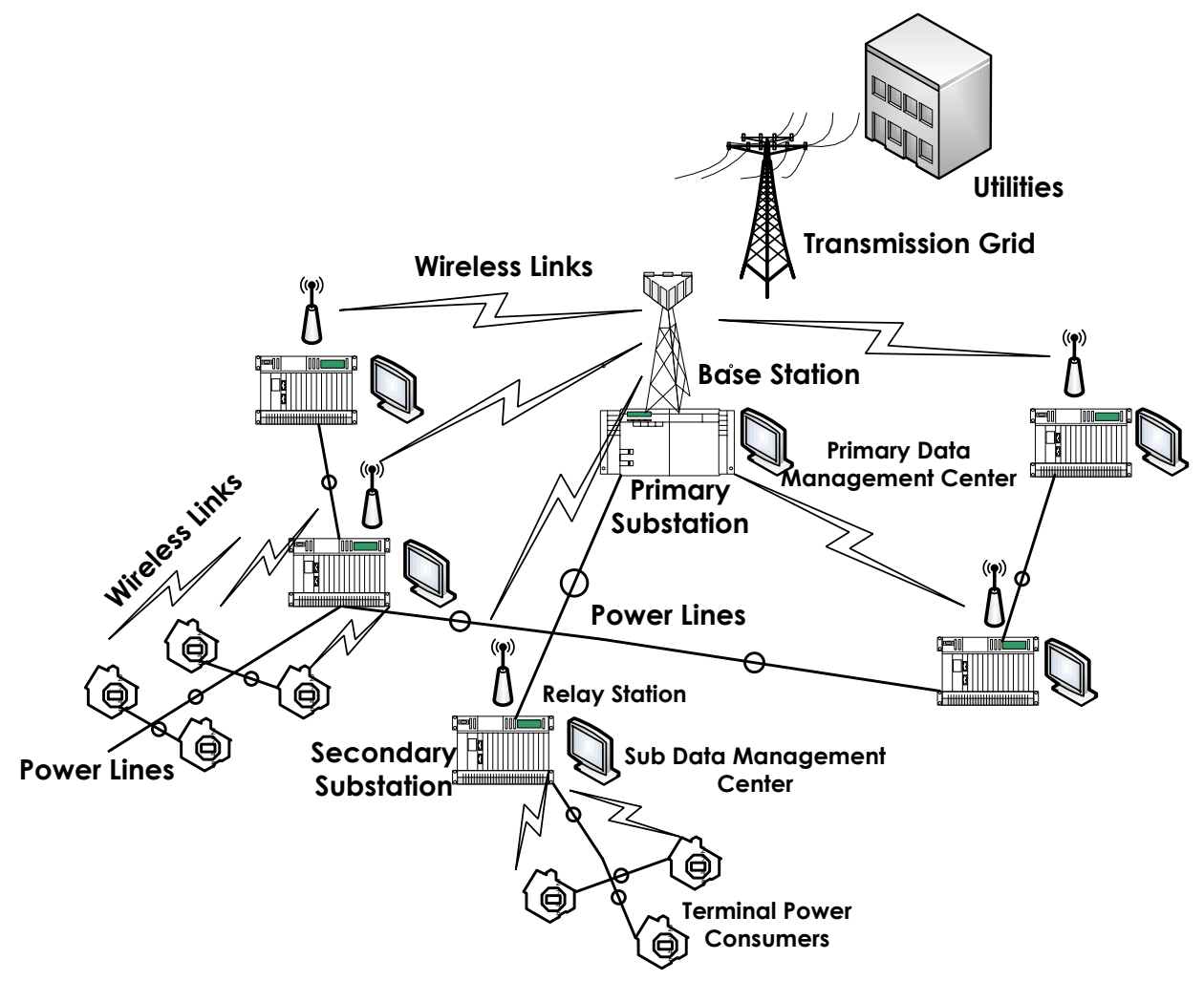

Fig. 2. The conceptual architecture of the distribution grid.

The considered TV spectrum is divided into $L$ channels : $\left\{C_{1}, C_{2}, \ldots, C_{L}\right\}$. The process of SG transmission based on CR technology is performed in three phases: spectrum sensing, channel selection, and data transmission. In the spectrum sensing phase, the idle channels are identified, and as a sequence, a channel (or more) is selected in the channel selection phase in order to transmit the data in the last phase. The following subsection provide additional details on each of the three phases.

\section{A. Phase I: Spectrum Sensing}

As mentioned earlier, the first phase is to identify the idle TV channel through the spectrum sensing process. The considered spectrum sensing technique in this work is energy detection, due to its simplicity and low cost [7]. Energy detection is performed by collecting a set of energy samples from each channel, 
and then comparing the accumulated average of the collected samples with a predefined threshold, denoted by $\lambda$. If the average is larger than than $\lambda$, the corresponding channel will be declared as used, while otherwise it will be identified as idle. The following formula describes the function of the detection process [8]

$$
d_{i}= \begin{cases}\text { used, } & \text { if } Y_{i} \geq \lambda \\ \text { idle, }, & \text { if } Y_{i}<\lambda\end{cases}
$$

where $d_{i}$ the detection decision of the $i^{\text {th }}$ channel, $Y_{i}$ is the average of the collected samples from the $i^{\text {th }}$ channel.

The performance of spectrum sensing is usually measured by two probabilities, namely, the detection probability $\left(P_{d}\right)$ and the false-alarm probability $\left(P_{f}\right)$. The detection probability is defined as the probability that a channel is identified as used given that it is used, and is expressed as follows [8]

$$
P_{d i}=Q\left(\frac{Y_{i}-\sigma_{n}^{2}-\sigma_{x}^{2}}{\frac{\sigma_{n}^{2}-\sigma_{x}^{2}}{\sqrt{S}}}\right)
$$

where $\sigma_{n}^{2}$ is the noise variance, $\sigma_{x}^{2}$ the variance of the transmitted signal, $S$ the number of the collected samples during the sensing, and $Q(x)=\frac{1}{\sqrt{2 \pi}} \int_{x}^{\infty} \exp \left(\frac{-t^{2}}{2}\right) \cdot d t$.

The false-alarm probability is the probability that a channel is identified as used given that it is idle, and is expressed as follows [8]

$$
P_{f i}=Q\left(\frac{Y_{i}-\sigma_{n}^{2}}{\frac{\sigma_{n}^{2}}{\sqrt{S}}}\right)
$$

The reader should note that high performance can be achieved by high detection probability and low false-alarm probability [9]. Moreover, it should be underlined that the spectrum sensing is imperfect process, i.e. the availability decision can be a false decision [10]. The false decision occurs in two cases, the false-alarm case and the missed-detection case. While the former is described earlier, the latter occurs when a channel is identified as idle given that is used. The missed detection is the complementary probability of the detection probability, and can be expressed as follows:

$$
P_{m d i}=1-Q\left(\frac{Y_{i}-\sigma_{n}^{2}-\sigma_{x}^{2}}{\frac{\sigma_{n}^{2}-\sigma_{x}^{2}}{\sqrt{S}}}\right)
$$

\section{B. Phase II: Channel Selection}

As the idle channel have been identified according to the spectrum sensing process, the transmitter node will be able to choose a channel for data transmission from the set of the available channels. The mechanism for selecting the used channel will be discussed later.

Generally, the achievable data rate of the $i^{t h}$ channel (in case it is actually idle) can be expressed as follows (Shannon's law):

$$
R_{i}=B \log _{2}\left(1+\gamma_{i}\right)
$$


where $B$ is the channel bandwidth and $\gamma_{i}$ is the signal to noise ratio of the $i^{t h}$ channel.

\section{Phase III: Data Transmission}

After identifying the transmit channel based on the adopted channel selection mechanism, the data transmission will start. The transmitted data can be classified into two categories as follows [15] [16] [17]:

- Delay Sensitive (DS): this type of data is very important and should be delivered as fast as possible to the control center. An example for this type is fault alarms.

- Non-Delay Sensitive (NDS): this type of data can tolerate a longer delay. For example, statistical data about the consumption, load variation, ..etc, as well as the regular meter readings.

\section{The Proposed Channel Selection Mechanisms}

In this section, we propose a channel selection mechanism for CR-base SG communications. The proposed mechanism is designed to adapt the selection criteria according to the type of data to transmit. Thus, it should (as will be proved later) provide high performance compared to other channel selection mechanisms.

The proposed channel selection mechanism is described in Fig. 3. The figure describes the whole process of SG communication based on CR technology, starting from spectrum sensing, channel estimation, channel selection, and data transmission.

As indicated in Fig. 3, the first step is to sense the channel set, which will result in identifying a subset of the sensed channels as idle. The idle subset can be different among nodes, since each node can experience different sensing performance and channel conditions. Therefore, the two communicating nodes should exchange the sensing results which include: $i$ ) the idle channel subset, and $i i$ ) the misseddetection probability of each channel in the idle channel subset. Notice that only channels that have been jointly (i.e. by both nodes) identified as idle can be used. The two missed-detection probabilities will be used to compute the average missed-detection probability of each channel in the joint idle channel subset. The average missed-detection probability is used to compute the reliability of the channel $\left(\rho_{i}\right)$ as follows

$$
\rho_{i}=\frac{1}{P_{m d i}}
$$

The main aim of the next step, channel estimation, is to estimate the SNR of each channel in the joint idle channel subset.

By performing the first two steps, the two communicating nodes will have $i$ ) a small subset of the channels that are commonly identified as idle, $i i$ ) the reliability value of each channel in the obtained subset, and iii) the SNR of each channel in the obtained subset. 
Now, once the transmitter node has data ready for transmission, the transmitter should examine the type of the transmitted data: i.e. "is it DS or NDS ?". According to the data type, the transmitter should select the proper channel. For DS data, the channel should be the most reliable channel, since DS data are very important and should be successfully delivered as soon as possible. On the other hand, for NDS data, the channel with highest SNR should be selected, since this type of data is large and requires high data rates.

The last step in the proposed mechanism is to ensure the delivery of the transmitted data, where we assume that the receiver node will inform the transmitter node if the data have been successfully delivered or not through an acknowledgment mechanism. If the data have not been successfully delivered, the transmitter node will re-transmit the data and exclude the previously used channel from the selection mechanism.

\section{Performance Analysis and Simulation Results}

In this section, the performance of the proposed channel selection mechanism is explored through simulation results. The results of other three channel selection mechanisms will be shown for comparison purpose. The three mechanisms are as follows:

- SNR-based Channel Selection Mechanism: it implies that the transmit channel will be selected based on the instantaneous SNR. Specifically, the channel whose SNR is the maximum will be selected from those that have been identified as idle by both the transmitter and the receiver.

- Reliability-based Channel Selection Mechanism: the most reliable channel is chosen for data transmission, where the channel whose instantaneous missed-detection probability is the minimum will be selected from channels tha have been identified as idle by both the transmitter and the receiver.

- Random Channel Selection Mechanism: unlike the above mechanisms, this mechanism randomly selects the transmit channel from the channels that have been identified as idle by both the transmitter and the receiver.

The reader should notice that the proposed mechanism can be considered as a combination of the first two mechanisms. In the proposed mechanism, the type of the transmitted data is used to identify the selection criteria (reliability or data rate) most appropriate for the data to be delivered. On the other hand, the three mechanisms described above do not explicitly consider the data type for channel selection process.

In the simulation setup, we consider the bandwidth of each channel equals to $20 \mathrm{KHz}$. The probability that a channel is occupied is identical for all channels and equals to 0.5 , which is a reasonable assumption, especially when no prior statistics about channels' usage are available. Regarding the sensing ability of the distributed nodes, the detection probability and the false-alarm probability are 


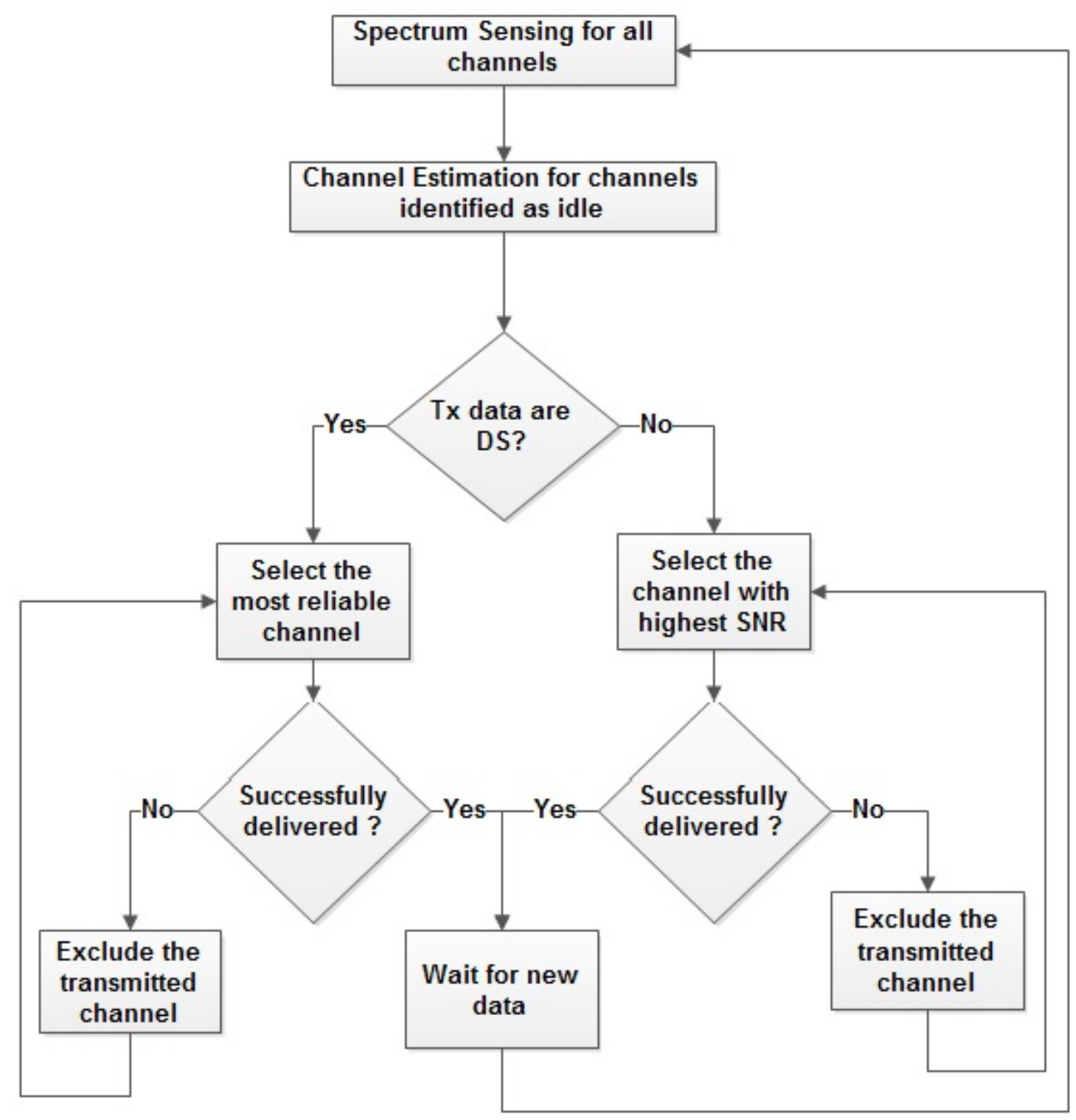

Fig. 3. The flow chart of the proposed channel selection mechanism for CR-based SG communications.

modeled as uniform random variables over the range $[0.5,1]$ and $[0,0.5]$, respectively. The probability that the transmitted data is delay-sensitive is 0.2 , while the probability of non-delay sensitive data is 0.8. Regardless of the employed mechanism, we consider that the transmitted data will not be delivered in two cases: $i$ ) if none of the channels has been identified as idle by both transmitter and receiver 
nodes, and $i i$ ) if the transmit channel is actually occupied by a primary user. The reader should notice that in case that the transmitted data have not been delivered, the transmitter node should retransmit the same data in the next transmission phase. The shown results are taken from $10^{4}$ transmission rounds.

In Fig. 4, the retransmission probability versus the total number of channels for the considered channel selection mechanisms is shown. As the proposed mechanism is the only one that distinguishes between the data type, it is plotted in Fig. 4 separately for delay-sensitive and non-delay-sensitive data types. The random and the SNR-based mechanisms show a poor performance whatever the channels' number due to not including the reliability of the transmit channel in the selection process. On the other hand, the reliability-based mechanism has the best performance, as it achieves the minimum retransmission probability. Regarding our proposed mechanism, it achieves the minimum retransmission probability (as in reliability-based mechanism) for only delay-sensitive data since this type of data cannot tolerate long delays. For non-delay-sensitive data, our proposal allows for higher retransmission probability (equals to the SNR-based mechanism).

The total achievable data rate versus the number of channels for all the considered mechanisms is shown in Fig. 5. Generally, for mechanisms that adopt the SNR in the selection (i.e. SNR-based and the proposed mechanism), the total data rate increases as the number of channels increases, while the other mechanisms (reliability-based and random mechanisms) achieve approximately constant total data rate although the number of channels is increasing. Intuitively, the SNR-based mechanism attains the maximum total data rate as it always chooses the maximum SNR for data transmission. However, the proposed mechanism is able to achieve high total data rate that is near to that achieved by the SNR-based mechanism. This is because the proposed mechanism partially (only for non-delay-sensitive data) depends on the channel SNR.

Comparing the results in Fig. 4 and Fig. 5, we can see that the reliability-based mechanism achieves the minimum retransmission probability, while it achieves low data rate. On the other hand, the SNRbased mechanism provides the maximum total data rate, while its achievable retransmission probability is high compared to the other mechanisms. In the light of this trade-off between reliability and data rate, the proposed mechanism achieves the balance for the trade-off, where it is able to achieve low retransmission probability for delay-sensitive data, and high data rate for non-delay-sensitive data.

The total data rate for all mechanisms versus the average SNR is shown in Fig. 6. Clearly, the total data rate increases for all mechanisms as the average SNR increases. The proposed mechanism achieves data rate that is near to the maximum value (achieved by the SNR-based mechanism).

In Fig. 7, the retransmission probability versus the occupancy probability of the channels for the proposed mechanism is depicted. The occupancy probability is the probability that the transmit channel is used by a primary user. It should be underlined that due to the fact that the proposed mechanism changes the selection criteria according to the data type, the delay-sensitive data can experience lower 
retransmission probability than the non-delay sensitive data.

\section{CONCLUSIONS}

This paper investigates the channel selection problem of cognitive radio based smart grid communications in the distribution section. SNR-based mechanisms can offer high data rate, while the cost is in longer delays due to not considering the reliability of the transmit channels. On the other hand, reliability-based mechanisms are able to shorten the transmission delay, and the corresponding data rate loss is high. The proposed mechanism is able to achieve balance in this trade-off as it adapts the selection criteria based on the data type. The proposed mechanism selects the most reliable channel for delay-sensitive data, and the maximum SNR channel for non-delay-sensitive data. Simulation results have proved the high performance of the proposed mechanism compared to the other mechanisms.

\section{REFERENCES}

[1] Y. Yan, et al.,"A survey on smart grid communication infrastructures: Motivations, requirements and challenges", IEEE Communications Surveys and Tutorials, vol. 15, no.1, pp. 5-20, 2013.

[2] VC Gungor, D. ahin, "Cognitive radio networks for smart grid applications: A promising technology to overcome spectrum inefficiency”, IEEE Vehicular Technology Magazine, vol. 7, no. 2, pp. 41-46, 2012.

[3] J. Mitola III, and GQ. Maguire Jr. "Cognitive radio: making software radios more personal." IEEE Personal Communications, vol. 6, no.4, pp. 13-18, 1999.

[4] C. Cordeiro, et al. "IEEE 802.22: the first worldwide wireless standard based on cognitive radios." First IEEE International Symposium on New Frontiers in Dynamic Spectrum Access Networks, DySPAN, 2005.

[5] T. Ycek, and H. Arslan, "A survey of spectrum sensing algorithms for cognitive radio applications." IEEE Communications Surveys \& Tutorials, vol. 11, no.1, pp. 116-130, 2009.

[6] D. Cabric, S.S. Mishra, and R. W. Brodersen. "Implementation issues in spectrum sensing for cognitive radios." IEEE Conference record of the thirty-eighth Asilomar conference on Signals, systems and computers, 2004.

[7] F.F. Digham, M.-S. Alouini, M.K. Simon, "On the energy detection of unknown signals over fading channels", IEEE Transactions on Communications vol. 55, no. 1, (2007) 2124.

[8] S. Althunibat and F. Granelli, "On the reduction of power loss caused by imperfect spectrum sensing in OFDMA-based cognitive radio access.” IEEE Global Communications Conference (GLOBECOM), 2012.

[9] Y. Gao and Y. Jiang, "Performance analysis of a cognitive radio network with imperfect spectrum sensing." IEEE Conference on Computer Communications Workshops INFOCOM, 2010.

[10] S. Althunibat, M. Di Renzo, and F. Granelli,"Towards energy-efficient cooperative spectrum sensing for cognitive radio networks: an overview.” Telecommunication Systems vol. 59, no. 1, pp. 77-91, 2015.

[11] A. Ghassemi, S. Bavarian, and L. Lampe, "Cognitive Radio for Smart Grid Communications." Smart Grid Communications (SmartGridComm), IEEE 2010.

[12] G. A. Shah, V. C. Gungor, and O. B. Akan, "A Cross-Layer QoS-Aware Communication Framework in Cognitive Radio Sensor Networks for Smart Grid Applications.” Industrial Informatics, IEEE Transactions on vol. 9, issue 3, pp. 1477-1485, 2013. 
[13] H. G. Wang, Y. Qian, and H. Sharif, "Multimedia communications over cognitive radio networks for smart grid applications." Wireless Communications, IEEE vol. 20, issue 4, pp. 125-132, 2013.

[14] J. F. Huang, H. G. Wang, Y. Qian, and C. G. Wang, ”Priority-Based Traffic Scheduling and Utility Optimization for Cognitive Radio Communication Infrastructure-Based Smart Grid.” Smart Grid, IEEE Transactions on vol.4, issue 1, pp. 78-86, 2013.

[15] W.P. Luan, D. Sharp, and S. Lancashire, "Smart Grid Communication Network Capacity Planning for Power Utilities." Transmission and Distribution Conference and Exposition, IEEE PES 2010.

[16] Y. Yan, Y. Qian, and H. Sharif, ”A Survey on Smart Grid Communication Infrastructures: Motivations, Requirements and Challenges," Communications Surveys \& Tutorials, IEEE vol. 15, issue 1, pp.5-20, 2012.

[17] A. Aggarwal, S. Kunta, and P. K. Verma, "A Proposed Communications Infrastructure for the Smart Grid," Innovative Smart Grid Technologies (ISGT) 2010. 


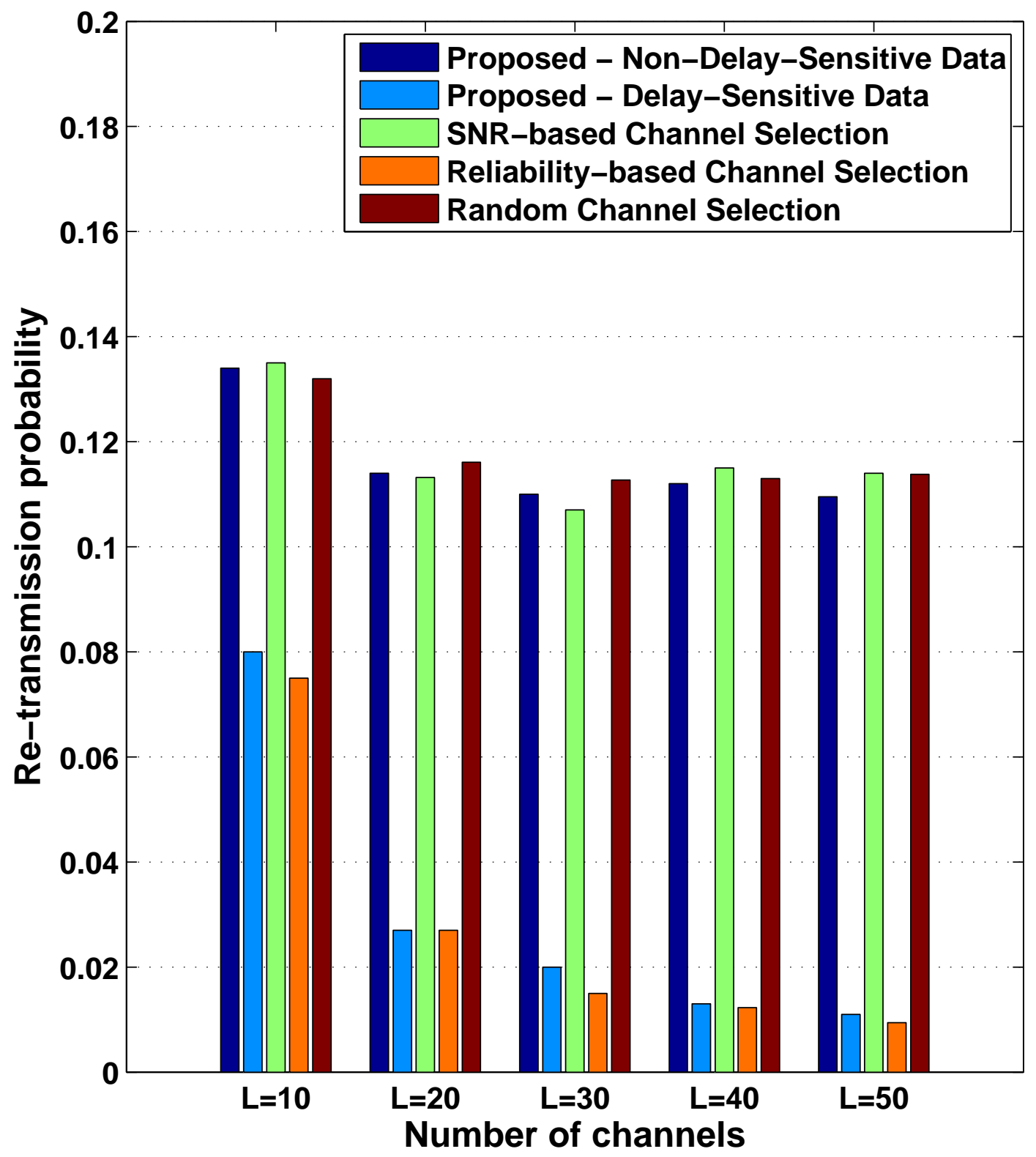

Fig. 4. The retransmission probability versus the total number of channels for the considered channel selection mechanisms. Average $\mathrm{SNR}=5$ 


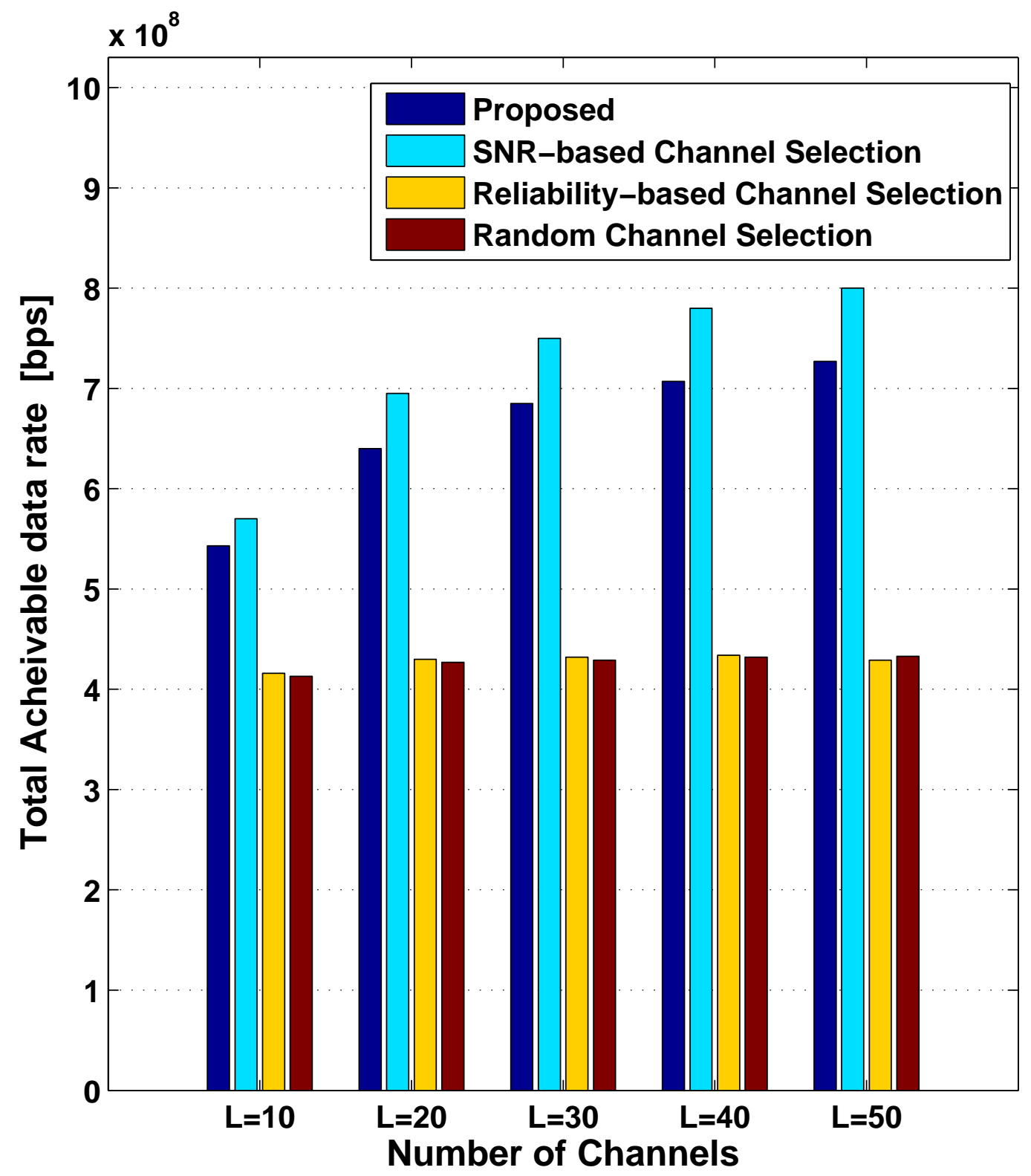

Fig. 5. The total achievable data rate versus the number of channels for all the considered mechanisms. Average SNR=5. 


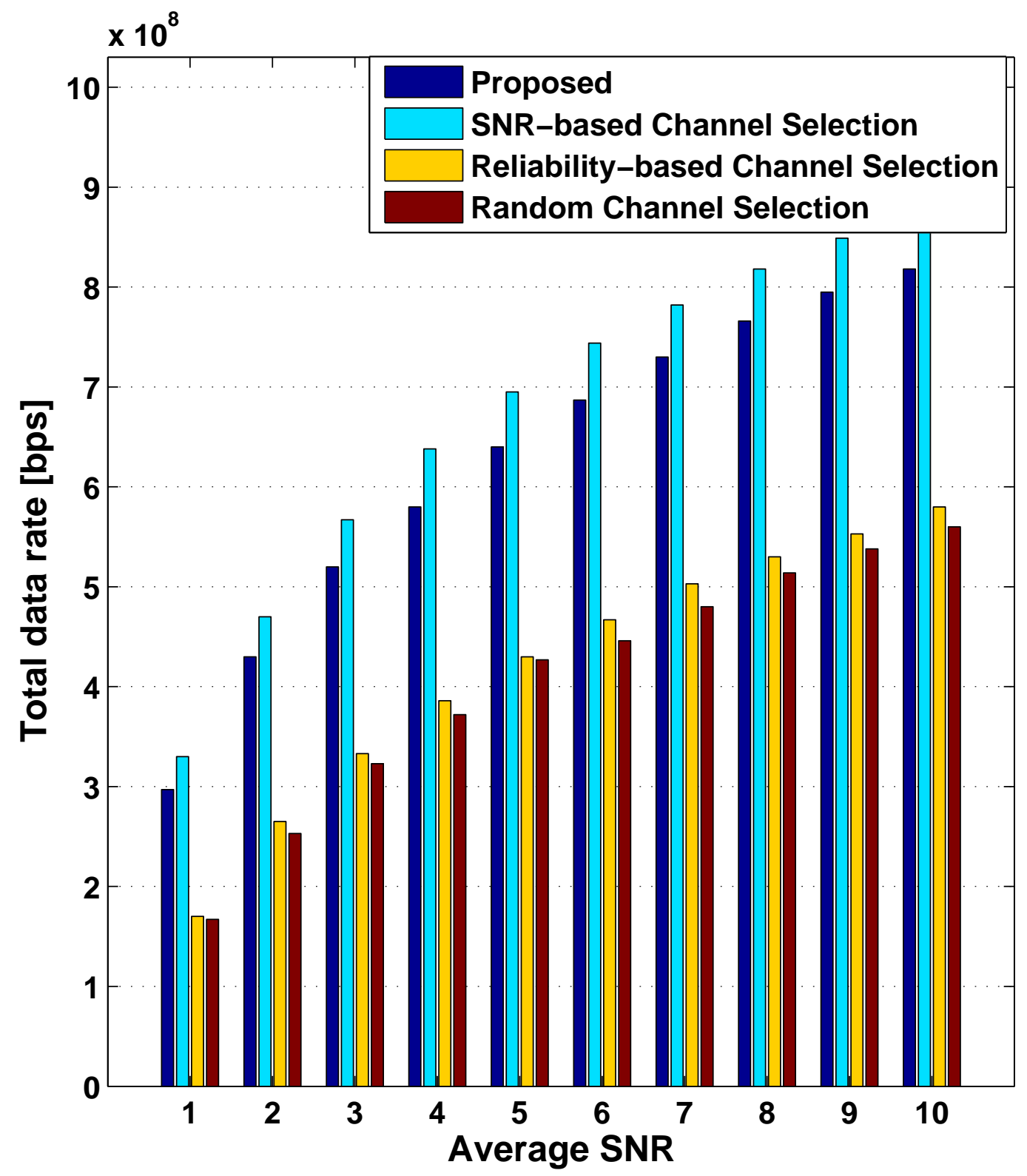

Fig. 6. The total data rate for all mechanisms versus the average SNR. Number of channels $=20$. 


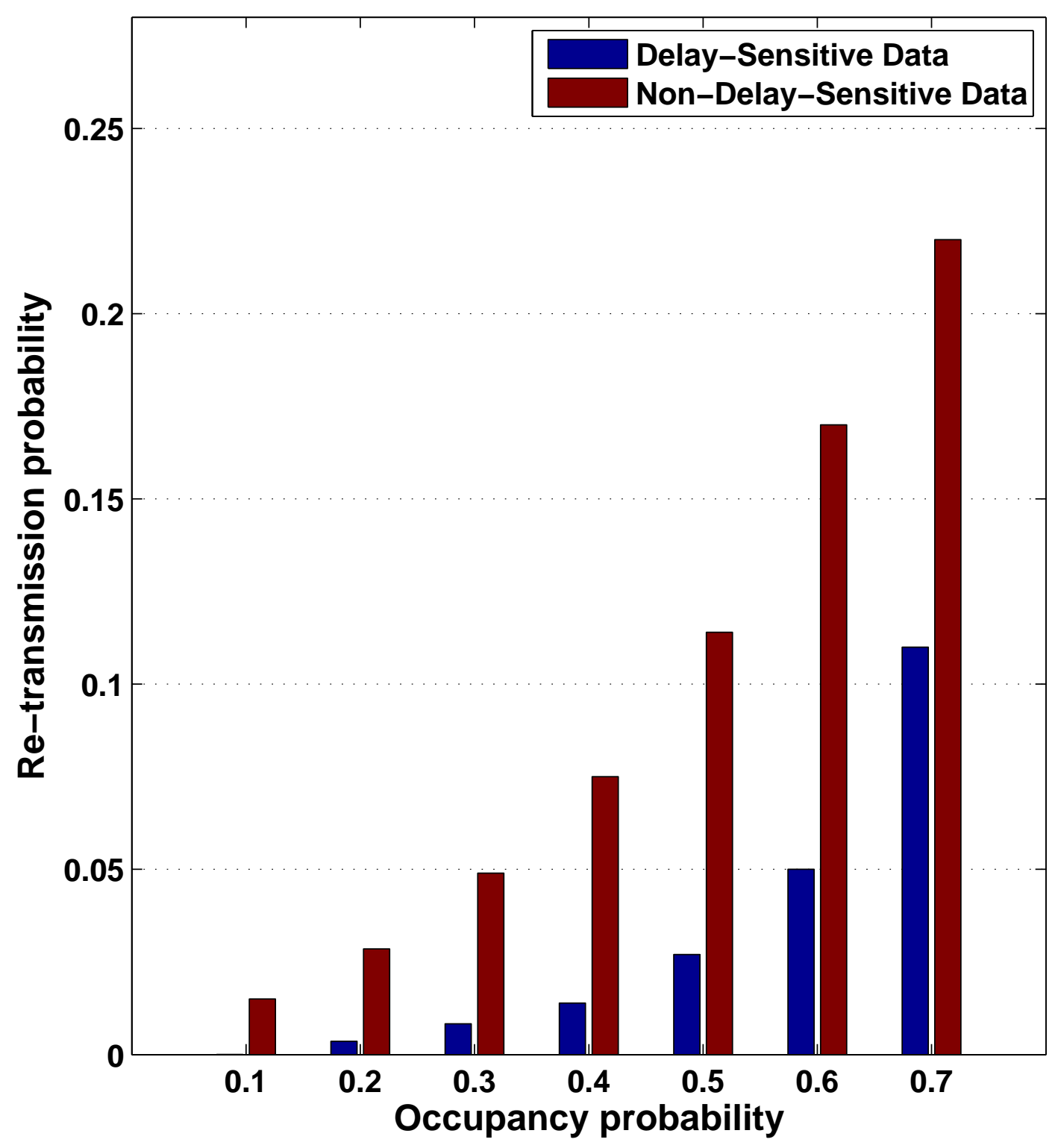

Fig. 7. The retransmission probability versus the occupancy probability of the channels for the proposed mechanism. Average $\mathrm{SNR}=5$, Number of channels $=20$. 